\title{
Gone, but not forgotten
}

Received wisdom holds that the notion of an 'aether' - a medium pervading all space, and the material 'substance' of the vacuum, if you will - was permanently relegated to the history books by Einstein's 1905 special theory of relativity, which showed it to be unnecessary, if not logically contradictory. But it seems, as it does so often in science, that the situation isn't quite as clear cut as that. Even Einstein had second thoughts on the matter. "I thought in 1905", he wrote in a paper in 1920, "that in physics one should not speak of the aether at all. This judgement was too radical...".

In 1951, writing in Nature, Paul Dirac argued that the concept of the aether had been dismissed prematurely. "If one re-examines the question in the light of present day knowledge", he wrote, "one finds that the aether is no longer ruled out by relativity, and good reasons can now be advanced for postulating an aether."

As Dirac pointed out, the principle of relativity demands that the perfect vacuum be isotropic in the sense of Lorentz, with all directions within the lightcone of any point being equivalent. The velocity of an aether would pick out one direction, and so violate this principle; hence, an aether with a definite velocity cannot be consistent with relativity. But quantum theory, Dirac argued, alters considerations of symmetry in an important way. A hydrogen atom, for example, can be in a spherically symmetric state, even though, considered in classical terms, a system of an electron orbiting a proton never could. Perhaps, he suggested, the vacuum could be similarly uncertain, spread over many velocities in a symmetric way, and so not violate the principle of relativity.

Dirac's thinking on this point hasn't restored the aether to mainstream physics, but it may have helped weaken the barrier against the exploration of such ideas, which persists today. Some extensions of general relativity, for example, postulate a timelike scalar field in addition to the spacetime metric. These so-called Einstein-aether theories effectively assert the existence of a preferred frame, defined by a dynamical field, which breaks local Lorentz invariance. This proposal has the advantage of being testable, as it predicts departures from standard

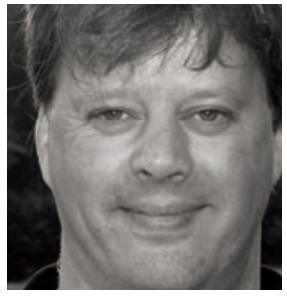

physics such as an inverse-square-law force acting between spins.

Other physicists have invoked an aether in trying to make sense of the 'spooky' action-at-a-distance apparently involved in quantum theory. In the spirit of playful speculation, for example, physicist Nicolas Gisin of the University of

The laws of physics haven't ruled out an aether with certainty. Geneva has explored what Bell-type experiments, measuring correlations between pairs of entangled particles, might imply about the velocity of quantum information. Given current experiments, he suggests, the velocity of such information would have to be greater than the speed of light, which can only make sense if there is a preferred frame, defined perhaps by the relative motions of the particles involved. No experiment, of course, has yet detected evidence for such a frame.

What do we learn from this? Perhaps that the laws of physics haven't ruled out an aether with quite the certainty many of us have been led to believe. But perhaps also that a concept so seductive and intuitively plausible is almost irresistible, and that the determined human mind can find a way to bring the idea into almost any theory.

Mark Buchanan

\section{The method of science}

Today, few would deny the central importance of science to our lives, but not many of the general public would be able to give a good account of what science is. To most, the word probably brings to mind not science itself, but the fruits of science - the pervasive complex of technology that has transformed all of our lives. However, science might also be thought to include the vast body of knowledge we have accumulated about the natural world. There are still mysteries, and there always will be mysteries, but the fact is that, by and large, we understand how nature works.

But science is even more than that. If one asks a scientist the question "what is science?", the answer will almost surely be that science is a process, a way of examining the natural world and discovering important truths about it. In short, the essence of science is the scientific method.

That stirring description suffers from an important shortcoming: we don't really know what the scientific method is. There have been many attempts at formulating a general theory of how science works, or at least how it ought to work, starting with Sir Francis Bacon. His idea, that science proceeds through the collection of observations without prejudice, has been rejected by all serious thinkers. Everything about

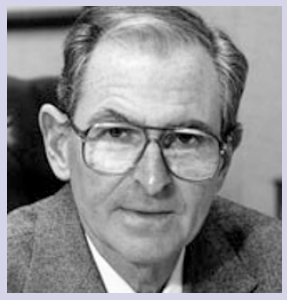

We don't really know what the scientific method is. the way we do science - the language, the instruments, the methods we use - depends on clear presuppositions about how the world works. Modern science is full of things that cannot even be observed at all. At the most fundamental level, it is impossible to observe nature without having some reason to choose what is worth observing and what is not worth observing. Once one makes that elementary choice, Bacon has been left behind.

Science, it turns out, is whatever scientists do. That, of course, is not an adequate philosophical description, but it may be the best we can do.

David Goodstein 